\title{
CONSUMER-RESOURCE BODY-SIZE RELATIONSHIPS IN NATURAL FOOD WEBS
}

\author{
Ulrich Brose,,${ }^{1,2,20}$ Tomas Jonsson, ${ }^{3}$ Eric L. Berlow,${ }^{1,2,4}$ Philip Warren, ${ }^{5}$ Carolin Banasek-Richter, ${ }^{1}$ \\ Louis-Félix Bersier, ${ }^{6}$ Julia L. Blanchard, ${ }^{7}$ Thomas Brey ${ }^{8}$ Stephen R. Carpenter, ${ }^{9}$ \\ Marie-France Cattin Blandenier, ${ }^{10}$ Lara Cushing, ${ }^{2}$ Hassan Ali Dawah, ${ }^{11}$ Tony Dell, ${ }^{12}$ Francois Edwards,${ }^{13}$ \\ Sarah Harper-Smith, ${ }^{14}$ Ute Jacob,${ }^{8}$ Mark E. Ledger,${ }^{13}$ Neo D. Martinez, ${ }^{2}$ Jane Memmott,${ }^{15}$ \\ Katja Mintenbeck,${ }^{8}$ John K. Pinnegar,${ }^{7}$ Buörn C. Rall, ${ }^{1}$ Thomas S. Rayner,${ }^{12}$ Daniel C. Reuman, ${ }^{16}$ \\ Liliane Ruess, ${ }^{17}$ Werner Ulrich, ${ }^{18}$ Richard J. Williams, ${ }^{2,21}$ Guy Woodward, ${ }^{19}$ and Joel E. Cohen ${ }^{16}$ \\ ${ }^{1}$ Department of Biology, Darmstadt University of Technology, Darmstadt, Germany \\ ${ }^{2}$ Pacific Ecoinformatics and Computational Ecology Lab, Berkeley, California 94703 USA \\ ${ }^{3}$ Systems Biology Group, School of Life Sciences, University of Skövde, Skövde, Sweden \\ ${ }^{4}$ University of California, Merced, Sierra Nevada Research Institute, Yosemite National Park, California 95389 USA \\ ${ }^{5}$ Department of Animal and Plant Sciences, University of Sheffield, Sheffield, UK \\ ${ }^{6}$ Department of Biology, Unit of Ecology and Evolution, Fribourg, Switzerland \\ ${ }^{7}$ The Centre for Environment, Fisheries and Aquaculture Science (CEFAS), Suffolk, UK \\ ${ }^{8}$ Alfred Wegener Institute for Polar and Marine Research, Bremerhaven, Germany \\ ${ }^{9}$ Center for Limnology, University of Wisconsin, Madison, Wisconsin 53706 USA \\ ${ }^{10}$ Zoological Institute, C.P. 2, Neuchatel, Switzerland \\ ${ }^{11}$ King Khalid University, College of Science, Department of Biology, Abha, Saudi Arabia \\ ${ }^{12}$ Department of Zoology and Tropical Ecology, James Cook University, Townsville, Australia \\ ${ }^{13}$ School of Geography, Earth and Environmental Sciences, University of Birmingham, UK \\ ${ }^{14}$ Department of Biology, Western Washington University, Bellingham, USA \\ ${ }^{15}$ School of Biological Sciences, Woodland Road, Bristol, UK \\ ${ }^{16}$ Laboratory of Populations, Rockefeller and Columbia Universities, New York, New York, 10021 USA \\ ${ }^{17}$ Institute of Soil Science, University of Hohenheim, Stuttgart, Germany \\ ${ }^{18}$ Department of Animal Ecology, Nicolaus Copernicus University, Torun, Poland \\ ${ }^{19}$ School of Biological and Chemical Sciences, Queen Mary University of London, UK
}

\begin{abstract}
It has been suggested that differences in body size between consumer and resource species may have important implications for interaction strengths, population dynamics, and eventually food web structure, function, and evolution. Still, the general distribution of consumer-resource body-size ratios in real ecosystems, and whether they vary systematically among habitats or broad taxonomic groups, is poorly understood. Using a unique global database on consumer and resource body sizes, we show that the mean bodysize ratios of aquatic herbivorous and detritivorous consumers are several orders of magnitude larger than those of carnivorous predators. Carnivorous predator-prey body-size ratios vary across different habitats and predator and prey types (invertebrates, ectotherm, and endotherm vertebrates). Predator-prey body-size ratios are on average significantly higher (1) in freshwater habitats than in marine or terrestrial habitats, (2) for vertebrate than for invertebrate predators, and (3) for invertebrate than for ectotherm vertebrate prey. If recent studies that relate body-size ratios to interaction strengths are general, our results suggest that mean consumer-resource interaction strengths may vary systematically across different habitat categories and consumer types.
\end{abstract}

Key words: allometry; body length; body mass; body-size ratio; food webs; parasitoid-host; predation; predator-prey.

\section{INTRODUCTION}

Manuscript received 1 February 2006; revised 8 May 2006; accepted 9 May 2006. Corresponding Editor: H. Hillebrand.

${ }^{20}$ E-mail:brose@bio.tu-darmstadt.de

${ }^{21}$ Present address: Microsoft Research Ltd., Cambridge CB3 0FB UK.
Body size, one of the most fundamental characteristics of an organism, is linked to physical activities, such as locomotion, dispersal, and space use (Peters 1983, Jetz et al. 2004), to biological rates such as growth, 
respiration, reproduction, and mortality (Peters 1983, Brown et al. 2004), to evolutionary patterns (Loeuille and Loreau 2005), and to population characteristics such as abundance and trophic height in a food web (Jennings et al. 2001, Cohen et al. 2003). These relationships have stimulated theories about how species population dynamics, interaction strengths, and food web structure could depend on consumer-resource body-size ratios (Yodzis and Innes 1992, Jonsson and Ebenman 1998, Emmerson and Raffaelli 2004, Brose et al. 2005a, Reuman and Cohen 2005, Woodward et al. 2005, Wootton and Emmerson 2005). Since the distribution of trophic links and interaction strengths in a community may reflect size constraints on who eats whom, the ratio of body sizes between predators and their prey may play an important role in explaining regularities in food web structure (Warren and Lawton 1987, Cohen et al. 1993) and stability (De Ruiter et al. 1995, Neutel et al. 2002).

Empirically, predators (excluding pathogens, parasites and parasitoids) tend to be larger than their prey (Warren and Lawton 1987, Cohen et al. 1993, Memmott et al. 2000), but it remains open whether the body-size ratios vary systematically among ecosystems or consumers types. For instance, if size constraints on feeding are related to habitat structure, the consumer-resource body-size ratios could differ between terrestrial and pelagic ecosystems. Here, we analyze the largest global database on consumer and resource body sizes compiled so far (Brose et al. 2005b). We present data on body-size ratios for predators, parasitoids, herbivores, and detritivores. Using data of carnivorous predators, we test for significant differences in predator-prey body-size ratios depending on habitat categories and the predator types.

\section{Methods}

We use average body masses (g; empirical measurements of mass $[n=13085]$ or allometric estimates of mass based on empirical measurements of length $[n=$ 3778]) of 16863 consumer-resource links in a global data set (Brose et al. 2005b). The body sizes were measured for the individuals engaged in the trophic interactions: sometimes these individuals are adults, sometimes larvae. If possible, predator life stages with different feeding interactions are separated in these data. Individual consumer species are included several times when linked to different resource species and vice versa. Here, "consumer" is used as a collective term that includes predators (consumers that kill living animal prey and subsequently consume all or most of it), parasitoids (consumers that live in or on and consume a living individual animal that is killed simultaneously or subsequently when the larval development ends and the consumers emerge), parasites (consumers that eat part of an individual animal without directly killing it), herbivores (consumers of living plants), and detritivores (consumers that eat dead plant and animal material). Our data include different consumer and resource types (invertebrates, ectotherm and endotherm vertebrates) across different habitat categories (terrestrial, lake, stream, and marine ecosystems; see Brose et al. [2005b] for an overview of the ecosystems studied). The Grand Caricaie food web has been sampled twice (spring and fall) under two treatments (mown and unmown) at two different locations (Brose et al. 2005b). To avoid pseudoreplication, we use just one of these samples: the fall sample of the mown treatment of the Scirpus lacustrisdominated locality (ScMown 2). To our knowledge this sample does not systematically deviate from the other samples. We did not include data of parasitic consumers due to the low number of cases $(n=2)$, or of consumers with unknown habitat types. The remaining data include predatory $(n=5156)$, parasitoid $(n=215)$, herbivorous $(n=930)$, and detritivorous $(n=218)$ consumers (i.e., four feeding types) from terrestrial $(n=2245)$, lake $(n=$ 1214), stream $(n=736)$, and marine ecosystems $(n=$ 2324). For these 6519 interactions, we calculated the base-10 logarithm of the ratio of the body masses of consumers $\left(M_{\mathrm{C}}\right)$ to resources $\left(M_{\mathrm{R}}\right)$, hereafter called $\log _{10}$ body-mass ratios (i.e., $\left.\log _{10}\left(M_{\mathrm{C}} / M_{\mathrm{R}}\right)\right)$. These $\log _{10}$ body-mass ratios were analyzed for significant differences among the feeding types (ANOVA).

The data for herbivorous and detritivorous feeding interactions were sampled in aquatic habitats only, whereas all data for parasitoid interactions come from terrestrial habitats. Only the predator-prey data spanned all habitat categories. Therefore, to explore differences in consumer-resource body-mass patterns among habitat categories and consumer types, we restricted the analysis to predator-prey interactions (see Plate 1). We excluded 53 outliers in the original data set with residuals that deviate more than three standard deviations from the residual mean (residuals $>$ 3 sigma). These outliers include trophic interactions between (1) trout and zooplankton (copepods or waterfleas), (2) seals and nematodes, and (3) whales and zooplankton (see Discussion).

We carried out three regressions in the remaining data set $(n=5103)$ : ordinary linear least-squares regressions of (1) $\log _{10}$ predator mass as a function of $\log _{10}$ prey mass, (2) $\log _{10}$ prey mass as a function of $\log _{10}$ predator mass, and (3) a major axis regression (type II regression) that treats the two variables symmetrically. For regression 1, normality of the residuals was rejected (Lilliefors test) but linearity and homoscedasticity were accepted, whereas for regression 2, all three assumptions of the linear regression method were rejected. Normality assumptions of the statistical model underlying regression 3 were also violated by data (Jarque-Bera and Lilliefors tests). All standard error estimates and $P$ values for regressions were therefore produced by resampling ten thousand times, with replacement, 5103 data points from the data set and computing the parameters each time.

Because the body-mass ratios are log-transformed, "mean body-mass ratios" that we report below for 


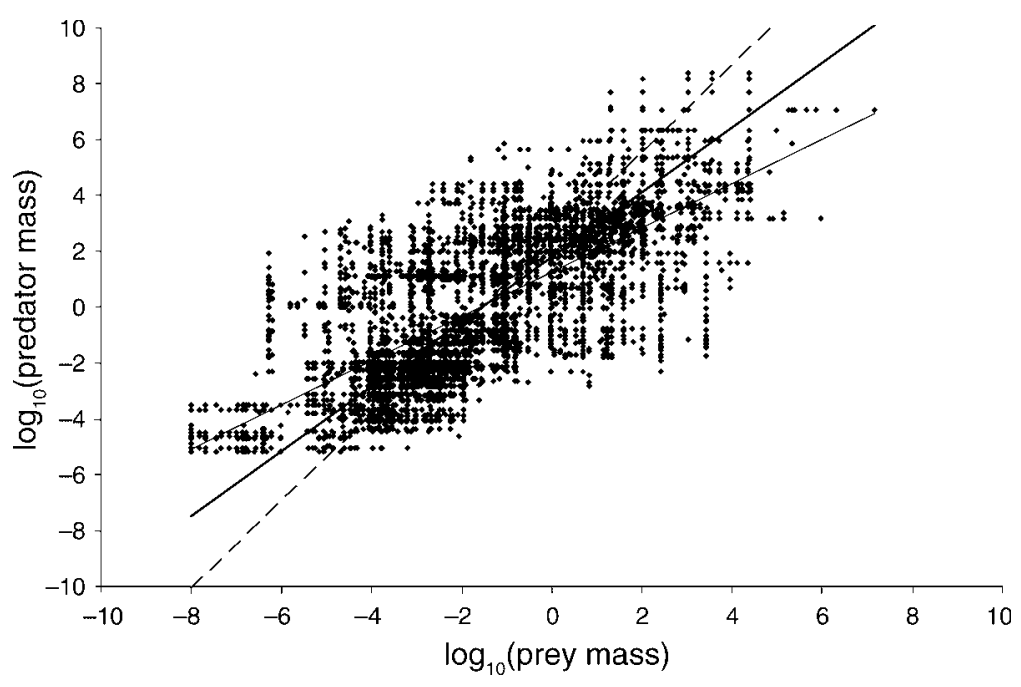

FIG. 1. Predator mass vs. prey mass. The major axis regression (heavy solid line, slope $=1.16 \pm 0.01$, intercept $=1.801, P<$ $0.0001, n=5103$ ) explains $86 \%$ of the variance in $\log _{10}$ predator mass. The other lines indicate the ordinary least-squares regressions of regression 1 ( $\log _{10}$ predator mass vs. $\log _{10}$ prey mass; thin solid line) and regression $2\left(\log _{10}\right.$ prey mass vs. $\log _{10}$ predator mass; dashed line, plotted with axes exchanged).

different habitat categories and predator and prey types are geometric means. Due to the absence of data on endotherm vertebrate predators from lakes and streams and the unequal number of replicates per cell, we used a type VI ANOVA with sigma-restricted parameterization. Our analyses focused on the characteristic bodymass ratios of trophic links, but because some species were engaged in more than a single trophic link, not all data points were strictly independent (see Discussion on caveats). To test the robustness of our results, we constructed a randomly sampled reduced data set, in which species occurred only once.

\section{Results}

Consumer-resource $\log _{10}$ body-mass ratios differ substantially among the feeding types $\left(F_{3,6515}=3525\right.$, $P<0.001)$. The consumer could be smaller than its resource $\left(\log _{10}\right.$ body-mass ratio $\left.<0\right)$, equally sized $\left(\log _{10}\right.$ body-mass ratio $=0)$, or larger $\left(\log _{10}\right.$ body-mass ratio $>$ $0)$. Predators are on geometric average about 42 times larger than their prey $\left(\log _{10}\right.$ body-mass ratio $=1.62 \pm$ 0.03 [mean $\pm \mathrm{sE}$ ], $n=5156$ ), whereas parasitoids are about three-quarters of the body size of their hosts $\left(\log _{10}\right.$ body-mass ratio $=-0.12 \pm 0.03, n=215$ ). The average $\log _{10}$ body-mass ratios of aquatic herbivores (8.99 \pm $0.15, n=930)$ and aquatic detritivores (13.41 $\pm 0.18, n=$ 218) are several orders of magnitude higher than those of aquatic predators $(1.82 \pm 0.04, n=3126)$.

Across all habitat categories and predator types, the predator mass and the prey mass increase together (Fig. 1). Both linear least-squares regressions yielded slopes significantly smaller than unity (regression $1, \log _{10}$ predator mass $=0.79 \times \log _{10}$ prey mass +1.26 ; regression $2, \log _{10}$ prey mass $=0.64 \times \log _{10}$ predator mass -1.53 ; standard error of slope $1=0.01$, standard error of slope $2=0.02, r^{2}=0.51, P<0.0001, n=5103$ ). The major axis regression has a slope of $1.16 \pm 0.01$ when $\log _{10}$ predator mass is plotted against $\log _{10}$ prey mass (Fig. 1). This coefficient suggests that the relative size difference (the $\log _{10}$ body-mass ratio) between predators and prey increases with increasing size of the species when data are pooled across all habitat categories and predator types. Three other regression methods symmetric in the variables (Babu and Feigelson 1992) also gave slopes significantly greater than 1 .

There was a significant interaction between predator type and habitat category when explaining variance in the $\log _{10}$ predator-prey body-mass ratios $\left(F_{4,5093}=\right.$ 29.542, $P<0.001$; Fig. 2). Relative size differences between predators and prey according to predator type are generally higher in freshwater habitats than in terrestrial or marine habitats, and habitat-specific differences exist for vertebrates but not for invertebrates (Fig. 2). These results are confirmed in the reduced data set, in which all species occur only once (predator type $\times$ habitat $F_{4,536}=4.6, P<0.01$ ). The average $\log _{10}$ bodymass ratios of invertebrate predators are generally smaller than those of ectotherm vertebrate and endotherm vertebrate predators (Table 1). A Scheffé post hoc test indicates no significant differences in average $\log _{10}$ body-mass ratios among (1) invertebrate predators in the different habitats, (2) lake and stream ectotherm vertebrates, and (3) terrestrial endotherm vertebrates and marine ecto- and endotherm vertebrates. All other groups are significantly different $(P<0.05)$. The $\log _{10}$ body-mass ratios also depend on the prey type. They are higher when invertebrates are consumed than when ectotherm vertebrates are eaten, for invertebrate predators (invertebrate prey, $0.66 \pm 0.03, n=2880$ vs. ectotherm vertebrate prey, $-0.46 \pm 0.29, n=35$ ), 


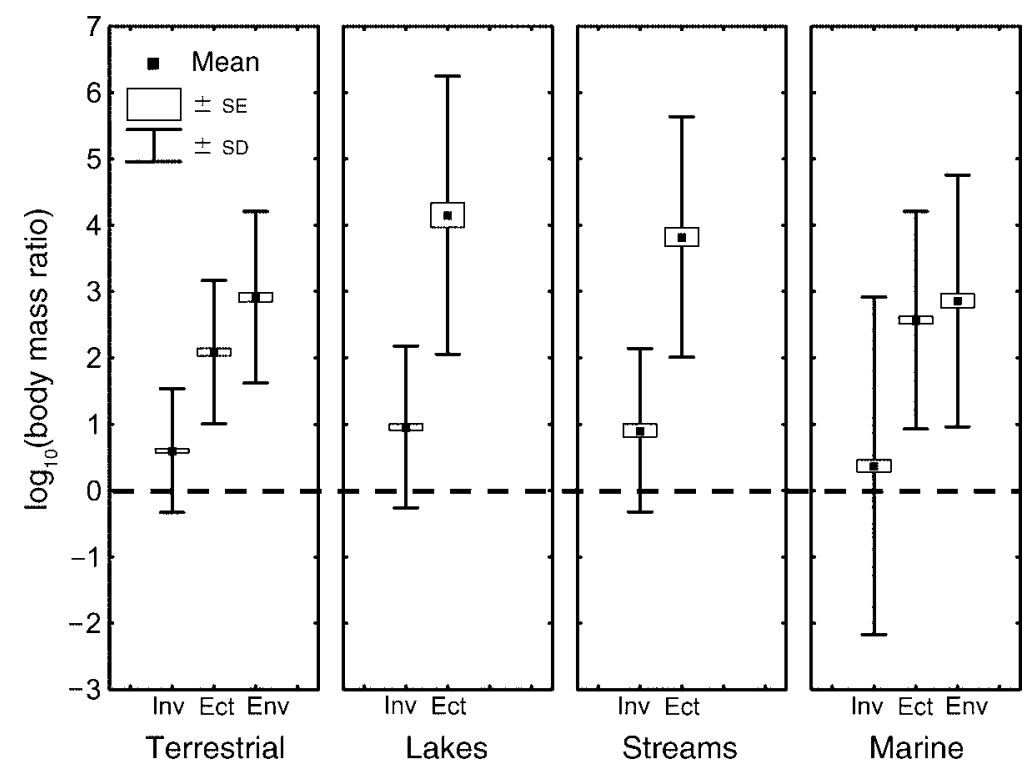

FIG. 2. Predator-prey body-size ratios (mean, SE, and SD) across the habitat categories and predator types (Inv, invertebrates; Ect, ectotherm vertebrates; Env, endotherm vertebrates). The dashed line indicates $\log _{10}$ body-size ratios of zero, which implies equally sized species. Data above and below the dashed line indicate predators that are larger and smaller than their prey, respectively.

ectotherm $(3.37 \pm 0.05, n=959$ vs. $1.55 \pm 0.05, n=$ $493)$, and for endotherm vertebrate predators $(3.54 \pm$ $0.07, n=401$ vs. $2.15 \pm 0.08, n=298)$.

\section{DISCUSSION}

We analyzed a large global database on the body masses of consumers and their trophic resources (Brose et al. 2005b). This database includes data from some previous studies of consumer-resource body-size patterns (Warren and Lawton 1987, Memmott et al. 2000), but adds recently published data, such as those of the Benguela system and Tuesday Lake, as well as previously unpublished data. In the following, we discuss our results for consumer-resource and preda-

TABle 1. Mean and standard error of the $\log _{10}$ body-mass ratio $\left(\log _{10}(C / R)\right)$, the $\log _{10}$ predator-mass $\left(\log _{10} C\right)$, and the $\log _{10}$ prey mass $\left(\log _{10} R\right)$ of the habitat and predator types.

\begin{tabular}{lcccr}
\hline \hline $\begin{array}{c}\text { Predator type } \\
\text { and habitat }\end{array}$ & $\log _{10}(C / R)$ & $\log _{10} C$ & $\log _{10} R$ & $n$ \\
\hline Invertebrate & & & & \\
$\quad$ Terrestrial & $0.6 \pm 0.03$ & $-2.64 \pm 0.02$ & $-3.24 \pm 0.02$ & 1263 \\
Lake & $0.96 \pm 0.05$ & $-1.93 \pm 0.05$ & $-2.89 \pm 0.08$ & 728 \\
Stream & $0.91 \pm 0.1$ & $-3.20 \pm 0.07$ & $-4.11 \pm 0.07$ & 165 \\
Marine & $0.37 \pm 0.09$ & $0.05 \pm 0.06$ & $-0.31 \pm 0.09$ & 759 \\
Ectotherm vertebrate & & & \\
Terrestrial & $2.08 \pm 0.06$ & $1.66 \pm 0.05$ & $-0.42 \pm 0.07$ & 375 \\
Lake & $4.15 \pm 0.18$ & $2.79 \pm 0.16$ & $-1.36 \pm 0.23$ & 130 \\
Stream & $3.82 \pm 0.14$ & $1.12 \pm 0.09$ & $-2.69 \pm 0.14$ & 175 \\
Marine & $2.57 \pm 0.06$ & $2.66 \pm 0.04$ & $0.09 \pm 0.07$ & 783 \\
Endotherm vertebrate & & & \\
Terrestrial & $2.91 \pm 0.07$ & $2.61 \pm 0.05$ & $-0.3 \pm 0.09$ & 392 \\
Marine & $2.86 \pm 0.1$ & $4.07 \pm 0.1$ & $1.21 \pm 0.12$ & 333 \\
\hline
\end{tabular}

tor-prey body-mass ratios, caveats and implications for food web structure and dynamics.

\section{Consumer-resource body-mass ratios}

Consistent with previous studies (Cohen et al. 1993, Memmott et al. 2000), our results show that predators are on average larger than their prey, whereas parasitoids are more similar in size to their hosts (Cohen et al. 2005). Furthermore, herbivores and detritivores from freshwater and marine habitats have body-mass ratios that exceed those of other consumers by several orders of magnitude. These links might differ in their dynamical behavior from predator-prey links, but the data available are limited (Brose et al. 2005b). In aquatic systems, many herbivores and detritivores are gape limited and ingest whole particles (e.g., fine particulate matter, unicellular algae). In contrast, terrestrial plants are sessile and their herbivores are free from size constraints on resource handling. It is thus likely that most terrestrial herbivores are much smaller than their plant resources since these herbivores are primarily invertebrates and these plants are primarily vascular. Additionally, the body size of basal species determines their intrinsic growth rates, and terrestrial producers are often large and have long generation times, whereas aquatic producers are generally small and have short generation times. This difference could affect the dynamics of the communities. Our database lacks terrestrial herbivore-plant and detritivore-detritus interactions, and to understand the structural and dynamic characteristics of terrestrial food webs, it would be useful to fill this gap. 


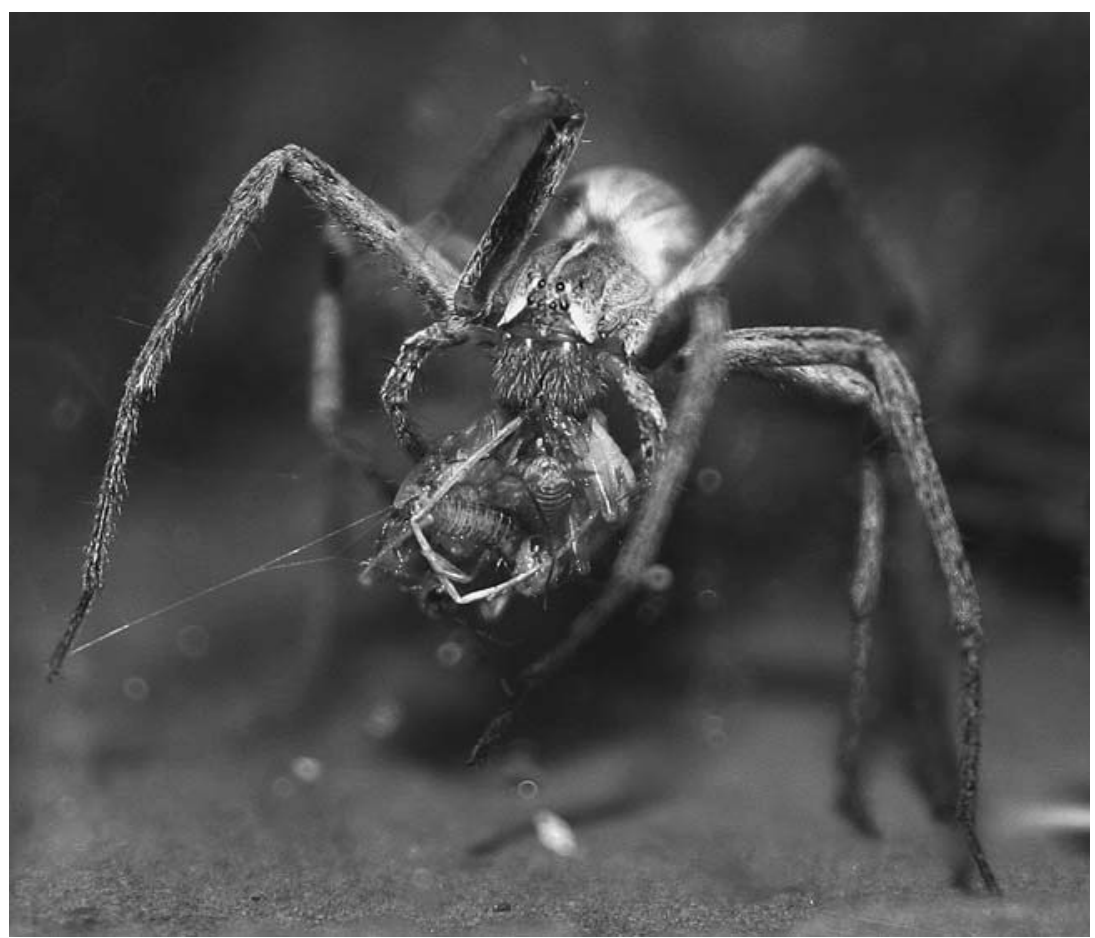

Plate 1. Predator-prey interaction between the lycosid spider Pisaura mirabilis and the cricket Gryllus assimilis. Photo credit: B. C. Rall.

\section{Predator-prey body-mass ratios}

Confirming previous analyses (Cohen et al. 1993), our results suggest that (1) most predators (80\%) are larger than their prey, and (2) predator size and prey size increase together. Our ordinary least-squares regression with predator size as the dependent variable had a slope significantly smaller than unity, suggesting that the size difference between predators and prey (the body-mass ratio) decreases with increasing prey size, as Cohen et al. (1993) found. Our ordinary least-squares regression with prey size as the dependent variable also had a slope smaller than unity suggesting that the body-mass ratio increases with increasing predator size. These results are mutually exclusive. However, they should both be discarded because standard linear models assume that the predictor variables were measured without error and that the distributions of residuals are normal, both tenuous assumptions for the data of this study. The major axis regression demonstrates that with increasing body sizes the size difference between predators and prey increases, in contrast to results of Cohen et al. (1993) based on ordinary linear regression. Woodward et al. (2005) show that the maximum prey size increases more strongly with predator size than the minimum prey size, so large predators feed on a larger size range of prey species and are less similar in size to their average prey size, as found here.

The geometric mean predator-prey body-mass ratio is substantially higher for vertebrate predators than for invertebrate predators, and it is lower for ectotherm vertebrate prey than for invertebrate prey. Do the bodymass ratios primarily depend on the predator type or on the prey type? Our data are too scarce for some combinations of predator and prey types (such as invertebrate predators and ectotherm vertebrate prey) to allow a full factorial analyses of this questions. Still, they suggest the predator type dominates: vertebrate predators have body-mass ratios to their prey that are more than two orders of magnitude larger than invertebrate predators for every prey type. Moreover, invertebrate predators have small body-mass ratios though they consume almost exclusively invertebrate prey, whereas vertebrate predators have high body-mass ratios even when they consume vertebrate prey. Therefore, we will subsequently focus on the predator types when discussing our results.

The body-mass ratios are higher for (1) vertebrate predators than invertebrate predators, and (2) ectotherm vertebrate predators in freshwater habitats than in terrestrial or marine habitats. As predator size increases 1.16 times as fast as prey size in our pooled data, higher ratios could reflect higher prey body-masses. The average prey size of invertebrates is roughly three and four orders of magnitude smaller than those of ectotherm and endotherm vertebrates, respectively. While this difference in prey size may account for an increase in body-mass ratios of $0.48(3 \times 0.16$, ectotherm vertebrates) and $0.64(4 \times 0.16$, endotherm vertebrates $)$, the actual differences in body-ratios between the 
predator types are more than two orders of magnitude. Moreover, the average prey size of ectotherm vertebrate predators is larger in terrestrial and marine habitats than in freshwater ecosystems, whereas their body-mass ratios are higher in the freshwater ecosystems. Differences in prey size can thus maximally account for a fourth of the differences in body-mass ratios between the predator types, and it is unlikely that they explain the observed differences between the habitat types.

Prey that are much smaller than the predator may contain too little energy to be worth the energetic costs of prey capture and consumption unless the prey can be harvested very efficiently by, for example, filter feeders such as baleen whales. Two of the groups of outliers that were removed (seal-nematode and whale-zooplankton interactions) represent this feeding strategy. By removing these links from the analysis we are not suggesting that these interactions are biologically unimportant. Rather these interactions represent a feeding strategy that is biologically interesting but cannot be described by the same body mass relationship as other predatorprey interactions. The large body-mass ratios of vertebrate predators indicate that they have optimized their ability to efficiently consume small bodied prey (vertebrate and invertebrate). At the other end of the scale, prey species that are very large relative to the predator will be difficult to capture and handle. Our results suggest that some invertebrate predators have optimized their morphology and physiology to allow effective handling of large bodied prey species (mainly invertebrates). For instance, the use of toxins and external digestion allows spiders to consume prey species of almost equal body size. Also, many invertebrate predators are suctorial (e.g., Hemiptera, some Diptera larvae, triclads, some leeches), which allows them to handle large prey.

The predator-prey body-mass ratios of vertebrate predators are generally higher in freshwater than in marine or terrestrial habitats in our data. The morphological constraints determining the maximum prey size are most important in pelagic ecosystems, where predators are restricted by the lack of hard surfaces. Here, most predators completely consume their prey (gape limitation) as they must avoid having their recently killed prey sink. This strong maximum size constraint on the interaction yields a clear size structure with high body-mass ratios in pelagic ecosystems, where energy flows from small phytoplankton to large predatory fish. Similar arguments apply to stream communities, where predators are often engulfers as they must prevent their prey from being swept away. Terrestrial predators also eat prey smaller than themselves, but in general, size constraints on trophic interactions are less pronounced than in aquatic habitats. Being supported by hard surfaces, terrestrial predators can successfully capture larger prey than their aquatic counterparts. While this difference in the physical structure of the environment may explain why terrestrial predators have smaller body-mass ratios than freshwater predators, the low body-mass ratios of marine invertebrate predators could result from a dominance of benthic species. Additional data from pelagic marine ecosystems and benthic lake ecosystems are needed to analyze systematically the possible differences between marine vs. freshwater and benthic vs. pelagic predator-prey body-mass ratios.

\section{Caveats}

Four caveats have to be recognized. First, the body sizes are averaged over the trophically interacting populations, but size-structured interactions may affect our analyses. In size-structured populations, if only large consumer individuals consume small resource individuals, these interactions are mischaracterized by ratios of averaged body masses. This caveat applies particularly to cannibalistic feeding interactions and consumers that change diet as they grow, which might be exemplified by one of the excluded groups of outliers (trout-copepod/ waterflea interaction). Second, the average body size of a resource population can sometimes describe the individuals that are not consumed instead of characterizing the trophic interaction. For instance, consumers might preferentially consume small resource individuals, thus increasing the average body size of the resource population. Third, under link-based approaches such as ours, several links share a common consumer or resource species. The data points are not independent in a statistically strict sense, thus violating the assumptions of sums of squares statistics (Reuman and Cohen 2004). While the first two obstacles can only be overcome by specific sampling procedures such as visual analyses of individual consumption events, the third point can be addressed by random subsamples of statistically independent data points (Warren and Lawton 1987). Following this approach, we have shown that our results hold in the reduced data set. Nevertheless, the $P$ values in the overall data set should be regarded as descriptive statistics, rather than probabilities (Reuman and Cohen 2004). Fourth, our data come from many distinct communities. The relationship between predator size and prey size across pooled communities need not be the same as that relationship in a given, local community. Further research is required to analyze the differences between a local community and an amalgam of multiple communities with respect to the relationship of predator and prey size.

\section{CONCLuSIONS}

Several data categories are absent or underrepresented in the data analyzed here (e.g., terrestrial detritivores and herbivores, invertebrates feeding on vertebrates). In addition to challenging other studies to corroborate or refute the findings reported here, we also call for new data on these consumer categories. Recent experiments suggest that the per capita interaction strength increases with the consumer-resource body-mass ratio (Emmer- 
son and Raffaelli 2004). If this relationship is general, our results suggest that the per capita effect of vertebrate predators on their prey could be higher on average than the per capita effect of invertebrate predators. Similarly, our predator-prey body-mass data suggest that per capita interaction strengths might increase from terrestrial to freshwater habitats and aquatic herbivores might have the strongest per capita impacts on their resources. These suggestions require testing.

\section{ACKNOWLEDGMENTS}

With regret we have had to remove the late Peter Yodzis from our list of authors. While we deeply appreciate the data and ideas that he contributed to this paper, his passing before having any chance to review the manuscript makes it unfair for us to assert his approval of its content by including him as an author. We thank Roland A. Knapp for providing data and Stefan Scheu for comments. Financial support has been provided to U. Brose, B. C. Rall, and C. Banasek-Richter by the German Research Foundation (BR 2315/1-1,2) and to J. E. Cohen and D. C. Reuman (DEB 9981552 and DMS 0443803) and to E. L. Berlow, L. Cushing, N. D. Martinez, and R. J. Williams (DBI-0234980 and ITR-0326460) by the United States National Science Foundation.

\section{Literature Cited}

Babu, G. J., and E. D. Feigelson. 1992. Analytical and MonteCarlo comparisons of 6 different linear least-squares fits. Communications in Statistics-Simulation and Computation 21:533-549.

Brose, U., E. L. Berlow, and N. D. Martinez. 2005a. Scaling up keystone effects from simple to complex ecological networks. Ecology Letters 8:1317-1325.

Brose, U., et al. 2005b. Body sizes of consumers and their resources. Ecology 86:2545.

Brown, J. H., J. F. Gillooly, A. P. Allen, V. M. Savage, and G. B. West. 2004. Toward a metabolic theory of ecology. Ecology 85:1771-1789.

Cohen, J. E., T. Jonsson, and S. R. Carpenter. 2003. Ecological community description using the food web, species abundance, and body size. Proceedings of the National Academy of Sciences (USA) 100:1781-1786.

Cohen, J. E., T. Jonsson, C. B. Müller, H. C. J. Godfray, and V. M. Savage. 2005. Body sizes of hosts and parasitoids in individual feeding relationships. Proceedings of the National Academy of Sciences (USA) 102:684-689.

Cohen, J. E., S. L. Pimm, P. Yodzis, and J. Saldana. 1993. Body sizes of animal predators and animal prey in food webs. Journal of Animal Ecology 62:67-78.
De Ruiter, P., A.-M. Neutel, and J. C. Moore. 1995. Energetics, patterns of interaction strengths, and stability in real ecosystems. Science 269:1257-1260.

Emmerson, M. C., and D. Raffaelli. 2004. Predator-prey body size, interaction strength and the stability of a real food web. Journal of Animal Ecology 73:399-409.

Jennings, S., J. K. Pinnegar, N. V. C. Polunin, and T. W. Boon. 2001. Weak cross-species relationships between body size and trophic level belie powerful size-based trophic structuring in fish communities. Journal of Animal Ecology 70:934-944.

Jetz, W., C. Carbone, J. Fulford, and J. H. Brown. 2004. The scaling of animal space use. Science 306:266-268.

Jonsson, T., and B. Ebenman. 1998. Effects of predator-prey body size ratios on the stability of food chains. Journal of Theoretical Biology 193:407-417.

Loeuille, N., and M. Loreau. 2005. Evolutionary emergence of size-structured food webs. Proceedings of the National Academy of Sciences (USA) 102:5761-5766.

Memmott, J., N. D. Martinez, and J. E. Cohen. 2000. Predators, parasitoids and pathogens: species richness, trophic generality and body sizes in a natural food web. Journal of Animal Ecology 69:1-15.

Neutel, A.-M., J. A. P. Heesterbeek, and P. C. De Ruiter. 2002. Stability in real food webs: weak links in long loops. Science 296:1120-1123.

Peters, R. H. 1983. The ecological implications of body size. Cambridge University Press, New York, New York, USA.

Reuman, D. C., and J. E. Cohen. 2004. Trophic links' length and slope in the Tuesday Lake food web with species' body mass and numerical abundance. Journal of Animal Ecology 73:852-866.

Reuman, D. C., and J. E. Cohen. 2005. Estimating relative energy fluxes using the food web, species abundance, and body size. Pages 137-182 in H. Caswell, editor. Advances in ecological research. Volume 36. Food webs: from connectivity to energetics. Academic Press, Elsevier, Burlington, Massachusetts, USA.

Warren, P. H., and J. H. Lawton. 1987. Invertebrate predatorprey body size relationships: an explanation for uppertriangular food webs and patterns in food web structure. Oecologia 74:231-235.

Woodward, G., B. Ebenman, M. Emmerson, J. M. Montoya, J. M. Olesen, A. Valido, and P. H. Warren. 2005. Body size in ecological networks. Trends In Ecology and Evolution 20: 402-409.

Wootton, J. T., and M. Emmerson. 2005. Measurement of interaction strength in nature. Annual Review of Ecology, Evolution, and Systematics 36:419-444.

Yodzis, P., and S. Innes. 1992. Body size and consumerresource dynamics. American Naturalist 139:1151-1175. 